\title{
Stellar Populations, Butcher-Oemler Effect, Star Formation in Clusters
}

\author{
Bianca M. Poggianti \\ Osservatorio Astronomico di Padova-INAF, Italy
}

\begin{abstract}
In this brief overview of the stellar populations of galaxies in clusters I highlight some of the most recent results, including the downsizing of the $\mathrm{k}+\mathrm{a}$ population and the star formation-local density relation. I conclude discussing some open questions regarding the interpretation of the observational results, and speculating on the current meaning of the words "primordial" and "environmental".
\end{abstract}

\section{Introduction}

Studying the evolutionary histories of galaxies in clusters has always been an endeavor to understand how galaxies (all galaxies) came to be as we observe them. In recent years, this quest can be better described as an effort to comprehend how galaxies formed and evolved in a "changing" environment. In fact, the common framework for these studies is now a hierarchical universe, in which galaxies can and often do change environment during their evolution. When a galaxy infalls into a cluster, it can do so as a single isolated galaxy, as a pair, as part of a group or as a member of another merging cluster. A galaxy can experience very different environmental conditions throughout its evolution. A major challenge for today's research is to identify the effects produced by the various environments on the observable properties of galaxies. It is especially hard to discriminate between minor and major influences. If all environments are potentially expected to have some impact on their galaxies, we would like to discern secondary effects ("cosmetics") from the primary causes that determine how galaxies are.

In this search we can rely on some objective and measurable properties, such as mass in stars and gas, their chemical content, their motion within the galaxy and the structures they design. The star formation activity is one of the most outstanding characteristics. Though a precise measurement of the current and past star formation activity is no easy task for any galaxy, star formation affects the accessible observables, such as colors and spectra, in such a manifest way that is often the most immediate evidence for evolution.

In this contribution I focus on the build-up of the stellar populations in cluster galaxies and on the evolution with redshift of their star formation activity. The subject has been very rich in results and papers published during the past 25 years, and it is impossible to give an exhaustive summary in a short text. I will therefore only touch upon some of the issues, and highlight just a couple of the most recent results. Overviews on the evolution of the stellar populations in cluster galaxies can be found in Ellingson (2003), Poggianti (2003a) and several re- 
views in the 3rd volume of the Carnegie Observatories Astrophysics Series: Clusters of Galaxies: Probes of Cosmological Structure and Galaxy Evolution (eds. J.S. Mulchaey, A. Dressler, and A. Oemler (Pasadena: Carnegie Observatories, http://www.ociw.edu/ociw/symposia/series/symposium3/proceedings.html).

\section{Where it all started....and where we are}

The Butcher-Oemler effect is the excess of galaxies bluer than the color-magnitude red sequence in clusters at $z>0.1-0.2$ as compared to the richest nearby clusters (Butcher \& Oemler 1984).

The origin of the scatter in the fraction of blue galaxies from cluster to cluster at any given redshift, and the consequent search for correlations between the blue fraction $f_{B}$ and the global cluster properties (Smail et al. 1998, Margoniner et al. 2001, Metevier et al. 2000); the trend with redshift of $f_{B}$ in optical and Xray selected cluster samples and the dependence on cluster selection (Andreon \& Ettori 1999, Ellingson et al. 2001, Kodama \& Bower 2001, Fairley et al. 2002); the change in galaxy colors with cluster-centric distance (Pimbblet et al. 2002); the nature of the galaxies giving rise to the Butcher-Oemler effect (De Propris et al. 2003); these are some of the aspects related to the Butcher-Oemler effect that are still subject of ongoing investigation, witnessing the interest in fully understanding the consequences and the cause of the seminal Butcher \& Oemler findings.

Put in a slightly different way, the Butcher-Oemler effect is the presence of large numbers of blue galaxies in rich clusters more distant than those in the local universe. This higher average level of activity in the past has been confirmed and greatly clarified by spectroscopic surveys and by Hubble Space Telescope morphological studies of galaxies in distant clusters (Couch et al. 1994, 1998, Dressler et al. 1997, Balogh et al. 1997, 1998, 1999, Poggianti et al. 1999, van Dokkum et al. 1999, 2000, Fabricant et al. 2000, Ellingson et al. 2001, Postman et al. 2001, Smail et al. 2001, Lubin et al. 2002).

No matter how the evolution in the populations of cluster galaxies is observed, if using the blue colors, the spectral features or the proportion of spiral versus early-type galaxies: all the three types of observations contribute to delineate a picture in which the evolution of galaxies in clusters is strong, given that a large fraction of them at $z=0$ have evolved from star-forming, late-type galaxies to passively evolving, early-type galaxies within a relatively short period of time (Kodama \& Smail 2001, van Dokkum \& Franx 2001).

At least three phenomena likely play a role in this evolution (Poggianti et al. 1999, Ellingson et al. 2001, Kodama \& Bower 2001): the declining infall rate of galaxies onto clusters at lower $z$ predicted by hierarchical cosmological models (Bower 1991, Kauffmann 1995); the evolution with $z$ of the average star formation rate (SFR) in the "field" galaxies that infall into clusters; and the decline of star formation in the cluster galaxies likely due to some physical process (or processes) that acts when galaxies infall in the denser environment. In fact, though the universe as a whole seems to evolve towards a progressively lower star formation activity in the Madau plot, such a trend appears to be accelerated in clusters (Kodama \& Bower 2001). 
The past star formation activity as seen from the absorption lines The most cited result obtained from spectra of galaxies in distant clusters is the presence of " $\mathrm{k}+\mathrm{a}$ " spectra, with no emission lines and strong Balmer lines in absorption indicating a vigorous star formation activity that was terminated at some point during the last 1-1.5 Gyr (Dressler \& Gunn 1983, Couch \& Sharples 1987). Since such spectra unequivocally identify post-starburst or post-starforming galaxies, their excess in distant clusters as compared to the field at similar redshifts is strong, if not the strongest, evidence that star formation is truncated by the dense environment (Dressler et al. 1999, but see Balogh et al. 1999 for a different view). $\mathrm{K}+\mathrm{a}$ galaxies have been thoroughly discussed elsewhere (e.g. Poggianti $2003 \mathrm{~b}$ and references therein), but it is worth highlighting here a very recent result from Tran et al. (2003). On the basis of the galaxy velocity dispersions and radii, these authors find that the descendants of the k+a's observed at $z \sim 0.8$ must be among the most massive early-type galaxies in clusters today, while the maximum luminosity and galaxy velocity dispersion of cluster $\mathrm{k}+\mathrm{a}$ galaxies decrease towards lower redshifts. Another result pointing in the same direction is presented in Poggianti et al. (2004), where we analyzed the luminosity distribution of $\mathrm{k}+\mathrm{a}$ galaxies in the Coma cluster: no $\mathrm{k}+\mathrm{a}$ as luminous as in the distant clusters is observed, while $\mathrm{k}+\mathrm{a}$ 's are found to be a significant fraction of the dwarf galaxy population.

The ongoing star formation activity as seen from the emission lines In the absence of a significant AGN ionizing radiation, emission lines are present in a spectrum only when there is ongoing star formation activity.

In agreement with the other photometric and spectroscopic signs of current activity, a significant fraction of even the most luminous galaxies in distant clusters have been observed to have emission lines indicating they are actively forming stars (Dressler et al. 1999, Postman et al. 2001), though the fraction of emission line galaxies remains lower in clusters than in the field at all redshifts.

Whether the star formation activity in clusters is enhanced in some of the infalling galaxies, and how relevant this is for the global cluster populations is still debated. Besides considerations based on optical observations in favor and against cluster-triggered starbursts, a striking result comes from dust-free wavelengths: the large numbers of Luminous Infrared starburst galaxies detected by ISOCAM in distant clusters seem to be too high to be consistent with simple accretion of starburst galaxies from the field.

Finally, several works have been recently devoted to the study of the emission line trend with clustercentric radius. Balogh et al. (1997, 1998) have shown how the mean equivalent width of $[\mathrm{O}$ II] increases radially in distant clusters, failing to reach the mean field value even as far out as several Mpc from the cluster centre. An extension of these radial studies has been carried out using the large datasets of the $2 \mathrm{dF}$ (Lewis et al. 2002) and Sloan (Gomez et al. 2003) spectroscopic surveys. As shown in Fig. 1, the mean $\mathrm{EW}(\mathrm{H} \alpha)$ increases at lower projected local surface density of galaxies (and, correspondingly, larger distance from cluster centre) down to a density of $\sim 1$ galaxy per $\mathrm{Mpc}^{2}$, and approaches the field value at $\sim 3$ times the virial radius of the cluster.

The mean EW, or SFR, used in these studies is the mean over all galaxies (with and without emission lines, of any morphological type), including the passive early-type galaxies that dominate the cluster cores and the highest density 

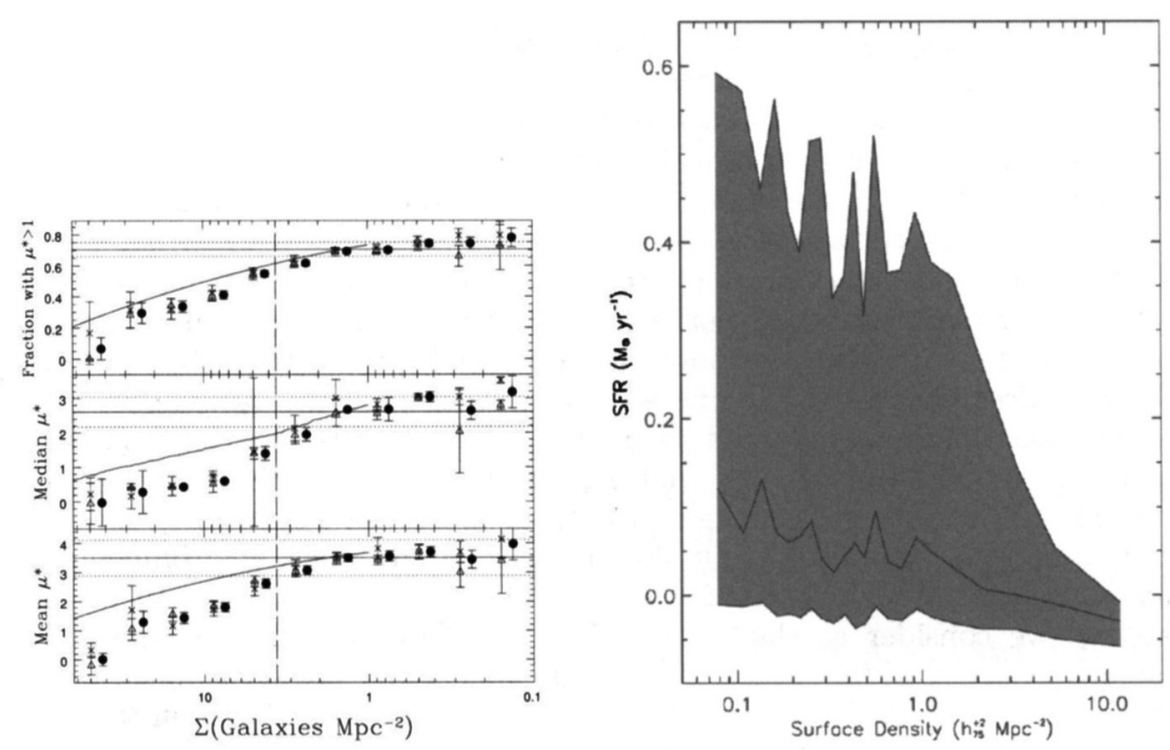

Figure 1. Left - From Lewis et al. $2002(2 \mathrm{dF}): \mu^{*}$, the star formation rate normalized to $L^{*}$, is proportional to the $\mathrm{EW}(\mathrm{H} \alpha)$. Its trend as a function of the local galaxy density is shown for all clusters (solid points) and for clusters with $\sigma$ greater than or less than $800 \mathrm{~km} \mathrm{~s}^{-1}$ (triangles and crosses, respectively). The horizontal solid line represents the field value. Right From Gomez et al. 2003 (Sloan): the shaded area represents the distribution of star formation rates, corrected for extinction. The median is shown as a solid line. The top of the shaded area is the $75^{\text {th }}$ percentile, while the bottom is the $25^{\text {th }}$ percentile.

regions. A mean total EW decreasing with density and towards the cluster centre is therefore not surprising in this respect. Whether the trend of mean EW simply reflects a different morphological mix as a function of local density, and whether it can be fully explained by the morphology-density relation (Dressler et al. 1997) is still unclear (Lewis et al. 2002, Gomez et al. 2003).

The origin of the observed EW trend remains in my opinion one of the most interesting questions to be answered, together with the origin of the morphologydensity relation and the relation between the two. A systematic variation of galaxy properties with the environment does not necessarily imply a "transformation" due to an environmental process beginning to act on a galaxy when this enters an environment for the first time. In principle, one cannot exclude that the correlation of star formation with local density can be partly or fully explained as an "imprinting" on the galaxy star formation history established in the early universe as a function of the local density. For example, the most massive ellipticals in the densest regions today (the cluster cores), that formed most of their stars at very high redshifts, were in the densest regions also at $z>3$ and it is then, by those conditions, that their star formation history was decided. These galaxies contribute to the shape of the EW-density relation today as some of the galaxies with $\mathrm{EW}=0$ (devoid of $\mathrm{SF}$ ), and it is therefore logical to conclude that at least part of the SF-density relation has been established very early on. 
Similarly, the morphology-density (MD) relation must have a "primordial" component, since at all redshifts the most massive early-type galaxies are found in the highest density regions. However, the MD relation as we observe it today is not fully established at high redshift, because we observe how it evolves in clusters, with late type spirals being transformed into early-type galaxies (Dressler et al. 1997, Lubin et al. 2002).

It is probably time for the astronomical community working on clusters to reconsider the meaning of words such as "primordial" and "environmental". By "primordial", do we mean anything that took place at $z>3$, or anything that was already engraved in the galaxy "destiny" by the early conditions in the location where they formed their first stars? And by "environmental", do we mean any physical process that affects galaxies once they enter a "new" environment, implying that their evolution would have been different if they did not become part of it? Or do we consider an effect environmental any time it has to do with something external to the galaxy itself? In this case, what do we consider as the "galaxy itself"? Anything that will be part of that galaxy by $z=0$ ? Can a process be considered an environmental effect even if it only affected the galaxy at $z>3$ ? Re-thinking the definition of the words "primordial" and "environmental" is not just a semantic exercise, it is probably useful to make progress in an era in which we believe galaxies can change environment, but today's environment is not necessarily unrelated with the environments of yesterday.

Acknowledgments. I thank the IAU and the organizers of this Joint Discussion on clusters for their kind invitation and for generously supporting my participation with a IAU travel grant.

\section{References}

Andreon, S., Ettori, S., 1999, ApJ, 516, 647

Balogh, M.L., Morris, S.L., Yee, H.K.C., Carlberg, R.G., Ellingson, E., 1997, ApJ, 488, L75

Balogh, M.L., Schade, D., Morris, S.L., Yee, H. K. C., Carlberg, R. G., Ellingson, E., 1998, ApJ, 504, L75

Balogh, M. L., Morris, S. L., Yee, H. K. C., Carlberg, R. G., \& Ellingson, E., 1999, ApJ, 527, 54

Bower, R.G., 1991, MNRAS, 248, 332

Butcher, H. \& Oemler, A., 1984, ApJ, 285, 426

Couch, W. J., \& Sharples, R. M. 1987, MNRAS, 229, 423

Couch, W. J., Ellis, R. S., Sharples, R.M., Smail, I. 1994, ApJ, 430, 121

Couch, W. J., Barger, A.J., Smail, I., Ellis, R. S., Sharples, R.M. 1998, ApJ, 497, 188

De Propris, R., Stanford, S.A., Eisenhardt, P.R., Dickinson, M., 2003, ApJ, 598, 20

Dressler, A., \& Gunn, J. E. 1983, ApJ, 270, 7

Dressler, A., Oemler, A., Jr., Couch, W. J., Smail, I., Ellis, R. S., Barger, A., Butcher, H., Poggianti, B. M., Sharples, Ray M., 1997, ApJ, 490, 577

Dressler, A., Smail, I., Poggianti, B. M., Butcher, H., Couch, W. J., Ellis, R. S., \& Oemler, A. 1999, ApJS, 122, 51

Ellingson, E., Lin, H., Yee, H. K. C., \& Carlberg, R. G. 2001, ApJ, 547, 609 
Ellingson, E., 2003, in Matter and Energy in Clusters of Galaxies, eds. S. Bowyer and C.-Y. Hwang, ASP Conf.Series vol.301, p.221

Fabricant, D., Franx, M., van Dokkum, P., 2000, ApJ, 539, 577

Fairley, B.W., Jones, L.R., Wake, D.A., Collins, C.A., Burke, D.J., Nichol, R.C., Romer, A.K., 2002, MNRAS, 330, 755

Gomez, P.L., Nichol, R.C., Miller, C.J., et al., 2003, ApJ, 584, 210

Kauffmann, G., 1995, MN, 274, 153

Kodama, T., \& Bower, R. G. 2001, MNRAS, 321, 18

Kodama, T., Smail, I. 2001, MNRAS, 326, 637

Lewis, I., Balogh, M., De Propris, R. et al., 2002, MNRAS, 334, 673

Lubin, L. M., Oke, J. B., \& Postman, M. 2002, AJ, 124, 1905

Margoniner, V.E., de Carvalho, R.R., Gal, R.R., Djorgovski, S. G., 2001, ApJ, 548, 143

Metevier, A.J., Romer, A.K., Ulmer, M. P. 2000, AJ, 119, 1090

Pimbblet, K.A., Smail, I., Kodama, T., Couch, W., Edge, A.C., Zabludoff, A.I., O'Hely, E., 2002, MNRAS, 331, 333

Poggianti, B. M., Smail, I., Dressler, A., Couch, W. J., Barger, A. J., Butcher, H., Ellis, R. S., \& Oemler, A., 1999, ApJ, 518, 576

Poggianti B.M., 2003a, Ap\&SS 285, 121

Poggianti B.M., 2003b, in Carnegie Observatories Astrophysics Series, vol.3: Clusters of Galaxies: Probes of Cosmological Structure and Galaxy Evolution (eds. J.S. Mulchaey, A. Dressler, and A. Oemler (Pasadena: Carnegie Observatories, http://www.ociw.edu/ociw/symposia/series/symposium3/proceedings.html).

Poggianti, B.M., Bridges, T.J., Komiyama, Y., Yagi, M., Carter, D., Mobasher, B., Okamura, S., Kashikawa, N., 2004, ApJ, 601, 197

Postman, M., Lubin, L. M., Oke, J. B. 2001, AJ, 122, 1125

Smail, I., Edge, A.C., Ellis, R. S., Blandford, R.D., 1998, MNRAS, 293, 124

Smail, I., Kuntschner, H., Kodama, T., Smith, G. P., Packham, C., Fruchter, A. S., \& Hook, R. N. 2001, MNRAS, 323, 839

Tran, K.-V.H., Franx, M., Illingworth, G., Kelson, D.D., van Dokkum, P., 2003, ApJ, 599,865

van Dokkum, P. G., Franx, M., Fabricant, D., Kelson, D. D., \& Illingworth, G. D. 1999, ApJ, 520, L95

van Dokkum, P. G., Franx, M., Fabricant, D., Illingworth, G. D., \& Kelson, D. D. 2000, ApJ, 541, 95

van Dokkum, P.G., Franx, M., 2001, ApJ, 553, 90 